\title{
Proteomics: the new era of microbiology
}

\author{
Marco Toscano, ${ }^{1}$ Roberta de Grandi, ${ }^{1}$ Lorenzo Drago ${ }^{1,2}$ \\ ${ }^{1}$ Laboratory of Clinical Microbiology, Department of Biomedical Sciences for Health, University of Milan; ${ }^{2}$ Clinical- \\ Chemistry and Microbiology Lab, IRCCS Galeazzi Institute - University of Milan, Italy
}

\begin{abstract}
Summary
Proteome refers to the entire complement of protein, expressed by a genome, cell, tissue or organism which is studied by proteomics. The aim of the present work is to review the main applications of proteomics in the clinical microbiology.
\end{abstract}

\section{Introduction}

Proteome refers to the whole complement of protein expressed by a genome, cell, tissue or organism and it is actually characterized by proteomics, or the study of complete set of proteins produced at a given time (14). In the last decades, characterization and differentiation of microbial proteome was performed by means of gel-based and gel-free protein separation methods. Numerous techniques for the analysis of proteome exist and they can be divided in two major groups (1): i) spectrophotometric methods, which comprise HPLC (High-performance liquid chromatography) and LC/MS (Liquid chromatography-mass spectrometry); ii) antibodies-based methods, which include ELISA (Enzyme-linked immunosorbent assay), immunoprecipitation, immunoelectrophoresis and western blot.

In the last years, new techniques have been developed to deepen the characterization of proteome and to date, next generation sequencing is commonly used to determine the amino acid

Correspondence:Marco Toscano, Laboratory of Clinical Microbiology, Department of Biomedical Sciences for Health, University of Milan, 20133 Milan, Italy.

E-mail: toscano.marco1@gmail.com

Key words: proteomics, microbiology.

Contributions: the authors contributed equally.

Conflict of interest: the authors declare no potential conflict of interest.

Received for publication: 15 February 2018.

Accepted for publication: 15 February 2018.

(C) Copyright M. Toscano et al., 2017

Licensee PAGEPress, Italy

Microbiologia Medica 2017; 32:7348

doi:10.4081/mm.2017.7348

This article is distributed under the terms of the Creative Commons Attribution Noncommercial License (by-nc 4.0) which permits any noncommercial use, distribution, and reproduction in any medium, provided the original author(s) and source are credited. sequence of proteins (or peptide) and infer protein conformation.

In clinical field, the study of bacterial proteins is of fundamental importance to understand the specific role of microorganisms, especially pathogenic ones, towards the human health, being able not only to detect the presence of bacteria in biological samples, but also to evaluate biochemical pathways in which they are involved.

Furthermore, the integration between metagenomics, transcriptomics, metabolomics and bioinformatics become essential for the development of new important instruments for improving modern molecular biology and medicine with the final purpose of strengthening diagnostic capacities.

The aim of the present work is to review the main applications of proteomics in the clinical microbiology, in order to highlight recent progresses in pathogen identification, study of antibiotic resistance mechanisms, development of new antimicrobial drugs and diagnosis of bacterial infections.

\section{Application of proteomics}

Mass spectrometry is an analytical technique based on the ionization of chemical compounds into charged molecules (12). Subsequently, the ratio of their mass to charge $(\mathrm{m} / \mathrm{z})$ is measured. The development of matrix assisted laser desorption ionization (MALDI) allowed the application of this technique also to large biological molecules, such as proteins, and to numerous scientific fields (12). Peptides are then converted into ions by addition or loss of one or more than one protons. Today, MALDI-TOF is commonly used in laboratories of clinical microbiology for the identification of pathogens isolated from human samples. Diagnosis of bacterial infections in body fluids is usually performed considering metabolic and biochemical profile of microorganisms that requires 24-48 h. However, the identification of clinical isolated requires rapid, reliable and cost-effective methods to initiate an appropriate antibiotic therapy, thus favoring the patient's outcome (12).

Numerous studies highlighted the ability of MALDI-TOF MS to surpass conventional diagnostic techniques in both speed and accuracy in the detection of blood and urinary infections $(5,6,9)$. Interestingly, comparing MALDI-TOF MS with the traditional methods of bacterial identification, the former requires minimal processing time and is able to identify pathogens from urine in the presence of more than two uropathogens (4).

A proteomic approach can be used also for the characterization of bacterial antibiotic resistance; in the last decades, the emergence of pathogens resistant to antibiotics became a serious concern as it decreases the efficacy of antimicrobial treatments, leading to therapeutic failure (13). Bacteria possess numerous mechanisms to be protected from the action of antibiotics, such as enzymatic modification of the antimicrobial molecule, presence of efflux pumps, reduced permeability to antibiotics and mutation of the antibiotic target site (13). The study of bacterial proteome may help in detecting the specific set of proteins involved in the micro- 
bial resistance to antibiotic molecules, improving therapeutic strategies to by-pass antimicrobial inefficacy and find new drug targets for the development of innovative antibiotics. Indeed, one of the most promising application of proteomics is the discovery of new antibacterial drugs, evaluating microbial specific targets on which antimicrobial agents may be effective (2). In detail, the characterization of bacterial proteomes can be useful to define a pathway-specific stimulus or a proteomic mark highlighting the inhibition of a given target or investigate if modified antibacterial molecules are still active against a specific bacterial target. In detail, bacteria grow in vitro with and without the specific antimicrobial molecule to test, under standardized conditions. Subsequently, the proteome of these microorganisms is analyzed evaluating eventual modifications in the protein-expression pattern, highlighting those proteins which expression is significantly altered (2).

The last application of proteomics in clinical field that should to be highlighted is the diagnosis of bacterial infections.

Traditional culture-based techniques may require several days to allow a sufficient bacterial growth and provide a positive identification of microbial pathogens. During this period, patients are subjected to a sub-optimal empiric antibiotic treatment, often making the treatment of the ongoing infection more difficult (11). Consequently, a precise and rapid diagnosis of bacterial infection is fundamental to contain the infection and save patients' life.

Proteomics may represent a valid help in the diagnostic process, allowing a quick diagnosis even in those infections in which patients do not show typical clinical signs or clear alterations of laboratory parameters. Generally, two different approach of proteomics can be performed in the clinical diagnosis of bacterial infections: i) investigating the presence of bacterial protein components in a biological sample (e.g. blood, cerebrospinal fluid, etc.); ii) analyzing the host's protein profile to evaluate which proteins show an altered expression following the bacterial infections.

Both approaches enable a clear and rapid diagnosis of bacterial infections, highlighting specific biomarkers to be used in the diagnostic process.

Jesse et al. (2010), for instance, determined reliable markers for distinguishing bacterial meningitidis (BM) from viral meningitidis (VM), analyzing the cerebrospinal fluid for a quantitative proteomic screening (7).

Authors used a 2D-DIGE protocol to compare subjects with $\mathrm{BM}$ and VM, identifying six potential human biomarkers specific for BM. In particular, low levels of soluble amyloid precursor protein alpha/beta $(\operatorname{sAPP} \alpha / \beta)$ and a high amount of glial fibrillary acidic protein (GFAP) have been detected as the main proteins to be used in the diagnosis of bacterial meningitis (7).

These findings are of great importance as they may improve early diagnosis of BM and lead to a more specific individual therapy.

Finally, one of the most interesting application of proteomics is the study of all proteins expressed by a microbial population, such as the gut microbiota. The human gastrointestinal tract is colonized by very diverse population of microorganisms that play pivotal roles in maintaining and improving human health (10).

It is known that when an intestinal dysbiosis occurs, numerous adverse effects on the host can arise, leading also to the onset of serious diseases such as cancers, metabolic disorders and inflammatory diseases (3). Interestingly, the host's response and gut eubiosis is modulated not only by bacteria but also by the intestinal bacterial protein pattern, which is defined metaproteome. In this context, metaproteomic may help in elucidating microbial functionality and understanding the specific role of intestinal bacteria in both healthy and disease conditions.

Individuals affected by Crohn's disease (CD), for instance, are characterized by an altered expression of specific bacterial proteins at intestinal level (8). In detail, a higher amount of outer membrane proteins potentially involved in immune response, and a lower amount of proteins involved in the production of short chain fatty acids (SFCAs) and mucin degradation have been observed in CD subjects if compared to healthy control (8).

Although today metaproteomic provides important information about the bacterial ecosystem under consideration, only the integration between metaproteomics, metagenomics, metatranscriptomics and metabolomics can deepen the study and characterization of microbiota, highlighting its real role towards the human health and its correlation with the host.

Moreover, it is of fundamental importance to integrate all these omics approaches with specific bioinformatic tools that can give a real biological meaning to the great amount of valuable data we obtain.

In conclusion, microbial proteomics is becoming essential in providing a powerful tool for translational applications, improving clinical diagnosis and antimicrobial therapy, as well as deepening the characterization of bacterial pathways and the correlations existing between microorganism and the human health.

\section{References}

1. Ahmad Y, Arya A, Gangwar A, et al. Proteomics in diagnosis: past, present and future. J Proteomics Genomics 2014;1:103.

2. Brötz-Oesterhelt H, Bandow JE, Labischinski H. Bacterial proteomics and its role in antibacterial drug discovery. Mass Spectrom Rev 2005;24:549-65.

3. Carding S, Verbeke K, Vipond DT, et al. Dysbiosis of the gut microbiota in disease. Microb Ecol Health Dis 2015;26:26191.

4. Ferreira L, Sánchez-Juanes F, González-Avila M, et al. Direct identification of urinary tract pathogens from urine samples by matrix-assisted laser desorption ionization-time of flight mass spectrometry. J Clin Microbiol 2010;48:2110-5.

5. Foster AGW. Rapid identification of microbes in positive blood cultures by use of the vitek ms matrix-assisted laser desorption ionization-time of flight mass spectrometry system. J Clin Microbiol 2013;51:3717-9.

6. Haigh JD, Green IM, Ball D, et al. Rapid identification of bacteria from bioMérieux BacT/ALERT blood culture bottles by MALDI-TOF MS. Br J Biomed Sci 2013;70:149-55.

7. Jesse S, Steinacker P, Lehnert S, et al. A proteomic approach for the diagnosis of bacterial meningitis. PLoS One 2010;5:e10079.

8. Kolmeder CA, de Vos WM. Metaproteomics of our microbiome - developing insight in function and activity in man and model systems. J Proteomics 2014;97:3-16.

9. La Scola B, Raoult D. Direct identification of bacteria in positive blood culture bottles by matrix-assisted laser desorption ionisation time-of-flight mass spectrometry. PLoS One 2009;4:e8041.

10. Lee PY, Chin SF, Neoh HM, Jamal R. Metaproteomic analysis of human gut microbiota: where are we heading? J Biomed Sci 2017;24:36.

11. Millar BC, Xu J, Moore JE. Molecular diagnostics of medically important bacterial infections. Curr Issues Mol Biol 2007;9:21-39.

12. Singhal N, Kumar M, Kanaujia PK, Virdi JS. MALDI-TOF mass spectrometry: an emerging technology for microbial identification and diagnosis. Front Microbiol 2015;6:791.

13. Vranakis I, Goniotakis I, Psaroulaki A, et al. Proteome studies of bacterial antibiotic resistance mechanisms. J Proteomics 2014;97:88-99.

14. Wilkins MR, Williams KL. The extracellular matrix of the Dictyostelium discoideum slug. Experientia 1995;51:1189-96. 